\title{
One Vessel Coronary Disease
}

National Cancer Institute

\section{Source}

National Cancer Institute. One Vessel Coronary Disease. NCI Thesaurus. Code C99997.

There was greater than or equal to $50 \%$ stenosis (reduction in cross-sectional area) in one coronary artery. (ACC) 